\title{
Synthesis and Application of Metal Doped Silica Particles for Adsorptive Desulphurization of Fuels
}

\author{
Bushra Jabeen, Uzaira Rafique ${ }^{\dagger}$ \\ Department of Environmental Sciences, Fatima Jinnah Women University, Rawalpindi 46000, Pakistan
}

\begin{abstract}
Petroleum a vital commodity affecting every aspect of 21st century. Toxicity and adverse effects of sulphur as catalyst in petroleum products is of great concern required development of techniques for desulphurization in compliance with the International standards. Installation of desulphurizing units costs over \$200 million per unit placing economic burden on developing countries like Pakistan. Present study analysis of commercial fuels (station petrol and jet fuel JP8) on gas chromatography-mass spectrometry (GC-MS) identified sulphur concentration of $19.94 \mathrm{mg} / \mathrm{L}$ and $21.75 \mathrm{mg} / \mathrm{L}$, respectively. This scenario urged the researcher to attempt synthesis of material that is likely to offer good adsorption capacity for sulphur. Following protocol of sol-gel method, transition metals $(\mathrm{Ni}, \mathrm{Cu}, \mathrm{Zn})$ solution is gelated with tetraethoxysilane (TEOS; silica precursor) using glycerol. Fourier transform infrared spectroscopy (FTIR) spectra revealed bonding of Zn-O, $\mathrm{Cu}-\mathrm{O}$, and $\mathrm{Ni}-\mathrm{O}$ by stretching vibrations at $468 \mathrm{~cm}^{-1}, 617 \mathrm{~cm}^{-1}$, and $468 \mathrm{~cm}^{-1}$, respectively. Thiophene and Benzothiophene mixed in n-heptane and benzene (4:1) for preparation of Model Fuels I and II, respectively. Each of silica based metal was applied as adsorbent in batch mode to assess the removal efficiency. Results demonstrated optimal desulphurization of more than $90 \%$ following efficacy order as $\mathrm{Si}-\mathrm{Ni}>\mathrm{Si}-\mathrm{Zn}>\mathrm{Si}-\mathrm{Cu}$ based adsorbents. Proposed multilayered (Freundlich) adsorption mechanism follows $\pi$-complexation with pseudo secnd order kinetics.
\end{abstract}

Keywords: Adsorption, Desulphurization, Fuel, Silica particles, Transition metals

\section{Introduction}

Sulphur and organo-sulphur compounds (e.g., alkylated derivatives like thiophenes and benzothiophenes) are integral part of crude oil and processed fuels. Concentration of elemental sulphur (350-500 ppm) represents $0.12-0.25$ weight\% of total sulphur compounds [1], whereas thiophenes constitute $50 \%-80 \%$ of sulphur content in fuels. These compounds pose a great challenge to oil refining industry because of their corrosiveness, toxicity and adverse effects on the environment [2]. Automotive and power generation industries aim at removal of refractory sulphur to obtain ultra-low content preferably down to $0.1 \mathrm{mg} / \mathrm{L}$ [1]. Alkylated derivatives can be removed through catalytic reduction with hydrogen and $\mathrm{H}_{2} \mathrm{SO}_{4}$ [3] by a process of hydro-desulphurization. However, alkyl groups at 4 or 6 positions offer steric hindrance [4] and thus are considered refractory. Furthermore, high temperature stability of thiophenes increases its tendency to accumulate [2] and render unsuitable for removal by hydro-desulphurization.

Muzic et al. [5] suggested adsorptive desulphurization as an alternative method for the attainment of ultra-low sulphur content. Thiolane and sulfolane have been adsorbed on clay from water by Kim [6]. Metal oxide composites [7] have also been applied for adsorptive desulphurization. Hernández-Maldonado and Yang [8] investigated copper, nickel, and zinc based zeolites for desulphurization of fuels. Metal nanoparticles stabilized on silica and alumina due to the large surface areas offer better applications in adsorption.

The present study is undertaken to employ greener and relatively less destructive methods to obtain sulfur-free fuel. For this purpose, metal doped silica particles using sol-gel method were synthesized. Further, the potential of these materials was explored in applicability for adsorptive removal of thiophene and benzothiophene as representatives of organo-sulphur compounds.

It is understood that silica serves as good substrate for doping of metals [9] and allows modification in properties of incorporated metals through controlled performance [10]. Tetraethylorthosilicate (TEOS) is used in the present investigation as precursor for synthesis of metal doped particles. TEOS is preferred due to its low cost (ten times cheaper) than tetramethylorthosilicate (TMOS).
cC (1) This is an Open Access article distributed under the terms of the Creative Commons Attribution Non-Commercial License (http://creativecommons. org/ licenses/by-nc/3.0/) which permits unrestricted non-commercial use, distribution, and reproduction in any medium, provided the original work is properly cited.
Received March 18, 2014 Accepted July 18, 2014

$\uparrow$ Corresponding author

E-mail: uzairaiqbal@yahoo.com

Tel: +92-51-9270050-57 (ext. 137) Fax: +92-51-9271168

Copyright (C) 2014 Korean Society of Environmental Engineers 


\section{Materials and Methods}

\subsection{Materials}

TEOS (Merck), zinc nitrate (GPR), copper nitrate (Sigma-Aldrich), nickel nitrate (AnalaR), glycerol (99\% Riedel-de Haen), $\mathrm{HNO}_{3}(65 \%$ Riedel-de Haen), $\mathrm{NH}_{3}$ (33\% AnalaR), n-heptane (99\% Labscan), thiophene (>99\% Sigma-Aldrich), 1-benzothiophene (Merck).

\subsection{Synthesis of Metal doped Silica Particles}

Transition metals (copper, nickel, and zinc) are loaded on the silica matrix using sol-gel method following protocol of Chao et al. [11] with slight modifications. In the present investigation, glycerol is attempted as templating agent. Glycerol is preferred for its high porosity [12] and less toxicity [13]. The adopted method is environment friendly, simple, efficient and works optimally at room temperature.

The general layout of synthesis procedure follows: each metal salt solution (1 molar) was hydrolyzed using $10 \mathrm{~mL}$ of TEOS as silica precursor and $5 \mathrm{~mL}$ of glycerol. Solution $\mathrm{pH}$ was adjusted to 1.5 by adding $0.125 \mathrm{M} \mathrm{HNO}_{3}$. The reaction mixture was kept for stirring for $3 \mathrm{hr}$ followed by drop wise addition of ammonia solution to condense gelatin at $\mathrm{pH}$ 8-9. Gel was filtered, washed with alcohol and aged in ethanol-water system for 48-72 hr.

The metal doped silica particles were obtained, oven dried and calcined at $500^{\circ} \mathrm{C}$ for $5 \mathrm{hr}$ in muffle furnace. The calcination treatment converts silica composite into metal oxide as confirmed by IR spectra, thus enhancing binding potential of particles for thiophene and benzothiophene. Similar effects of heating are reported by Seredych et al. [14].

\subsection{Characterization of Metal Doped Silica Particles}

The synthesized particles were characterized for surface functional groups, crystallinity, particle size and morphology by standardized analytical techniques. Fourier transform infrared spectroscopy (FTIR) spectra of the pressed $\mathrm{KBr}$ pellets were recorded on Fourier transform infrared spectrophotometer (FTIR 8400; Shimadzu, Kyoto, Japan) from $4,000 \mathrm{~cm}^{-1}$ to $400 \mathrm{~cm}^{-1}$. The samples surface morphology and size was determined by scanning electron microscopy (SEM) coupled with EDX JED-2300 analysis station on 6490(LA) JEOL Machine with gold sputtering at a potential of $20 \mathrm{kV}$.

\subsection{Preparation of Fuel Samples}

Two fuel samples (coded as Model Fuel I and II) were prepared following protocol of Dai et al. [15]. Each fuel sample comprising of $80 \%$ n-heptane (aliphatic), 20\% benzene (aromatic), and 2,000 mg/L of organo-sulphur compound. Model Fuel I and II contains thiophene and benzothiophene, respectively.

Two commercial fuels (petrol and jet fuel JP8) samples were diluted in n-hexane and run on gas chromatography-mass spectrometry (GC-MS; GC-QP5050, Shimadzu) to determine the background concentration of sulphur in each fuel samples. The sample was injected onto a system and a split/splitless injector on DB-5 column (30 m, $0.25 \mathrm{~mm}$, I.D. $0.25 \mu \mathrm{m}$ film thickness). Oven was heated from $70^{\circ} \mathrm{C}-240^{\circ} \mathrm{C}$ at the rate of $5^{\circ} \mathrm{C} / \mathrm{min}$ under helium atmosphere. Electron ionization detector at $70 \mathrm{eV}$ was used with scanning range of 35-425 amu [16].

\subsection{Procedure for Batch Adsorption Experiment}

Assessment of adsorptive desulphurization was determined in a series of batch adsorption experiments. For each batch, a known mass $(2 \mathrm{mg} / \mathrm{kg}$ ) of metal doped silica particles was subjected to $2 \mu \mathrm{g} / \mathrm{L}$ of organo-sulphur compound. The mixture was agitated at $100 \mathrm{rpm}$. At regular intervals, an aliquot of sample was drawn and absorbance was noted on UV-visible spectrophotometer (UV-Vis 1601, Shimadzu) against $\lambda$ max of thiophene and benzothiophene at $231 \mathrm{~nm}$ and $297 \mathrm{~nm}$, respectively.

The concentration of organo-sulphur compounds adsorbed on each of the synthesized material was determined from standard calibration curves. Removal (in percentage) was calculated using the formula,

$$
\% R=\left(\frac{C_{i}-C_{t}}{C_{i}}\right) 100
$$

\section{Results and Discussion}

Metal doped silica particles are applied for adsorptive desulfurization of selected organo-sulphur compounds. To understand the mechanism of adsorption, the synthesized materials were also characterized using standardized techniques. Each sample was subjected to atomic force microscopy to evaluate surface topography, particle size, and surface roughness.

The micrographs (Fig. 1) depicts histogram height of 5.62, 1.93, and $2.25 \mathrm{~nm}$ with particle size of 5.2, 1.8, and $1.8 \mathrm{~nm}$ per division for silica doped with $\mathrm{Ni}, \mathrm{Cu}$, and $\mathrm{Zn}$, respectively. The sequence of particle size seems to follow the order of atomic number position in the periodic table that is $\mathrm{Ni}$ is larger than $\mathrm{Cu}$ and $\mathrm{Zn}$. It may be attributed to the larger atomic radii of former (175 pm) than later $(128 \mathrm{pm})$.

\subsection{SEM/EDX}

SEM images of nickel silica 32.98-52.15 nm, copper silica 111-318 $\mathrm{nm}$, and zinc silica 48.67-282.38 nm were recorded (Fig. 2).

The presently synthesized $\mathrm{Ni}, \mathrm{Cu}$, and $\mathrm{Zn}$ doped silica particles are larger than reported alumina mesoporous silica [11]. The particle size for all the synthesized silica based metal particles fall in the nano-size range confirming that these materials can be reported as nanoparticles. On the other hand, the different sized particles within the same sample matrix indicate polydispersity. The amorphous nature of materials contributes to polydispersity. This is significant in the application of these materials as adsorbents by providing a range of particle size to volume ratio for effective adsorption. The EDX results showed that all silica based metal nanoparticles contain $2.6 \%$ doped metal.

The Ni/Si ratio reported in literature [17] is relatively higher in comparison to $\mathrm{Ni}$ doped silica synthesized in present investigation. The lower nickel to silica ratio suggests that binding is preferably through silanol groups rather than metal coordination linkage. This proposed mechanism is also supported by the FTIR 

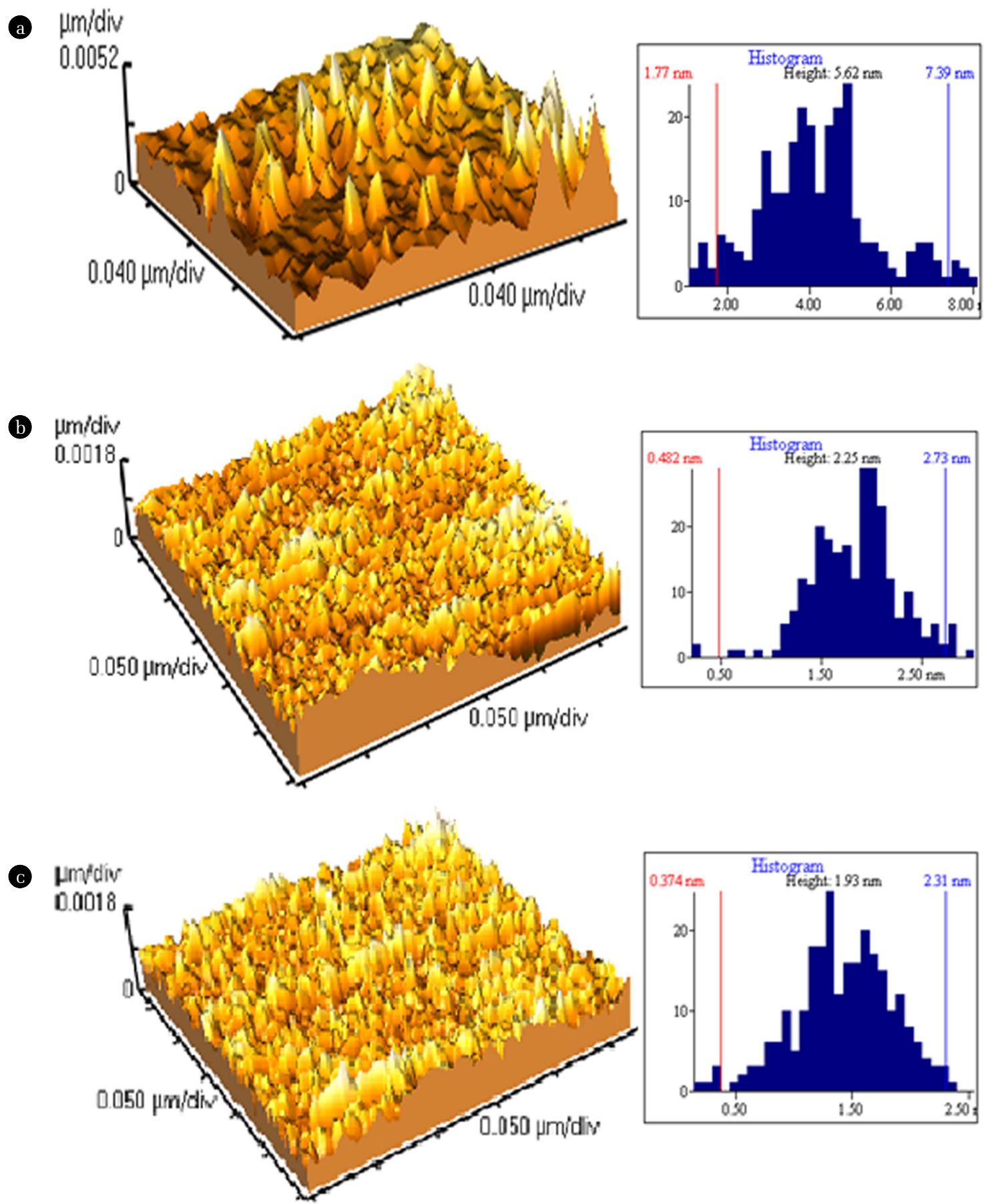

Fig. 1. Atomic force microscopy (AFM) images and histograms of silica doped (a) Si-Ni, (b) Si-Zn, and (c) Si-Cu particles.

analysis. The application of Ni doped silica particles in desulphurization of fuels is significant contribution of present investigation and is not reported elsewhere.

\subsection{X-Ray Diffraction Analysis}

The diffraction pattern of the copper doped silica particles yield
20: 35.2, 38.7, 48.7, 58.1, 61.3, 65.7, 68.1. The present experimental results are in agreement with those reported by Das et al. [18]. These values can be assigned to the reflection lines of monoclinic $\mathrm{CuO}$ particles [19] (Fig. 3).

The peak at $37.9^{\circ}$ corresponds to $\mathrm{NiO}$ [20], while the peak at $52.1^{\circ}$ corresponds to $\mathrm{Ni}$ species incorporated in to the silica 

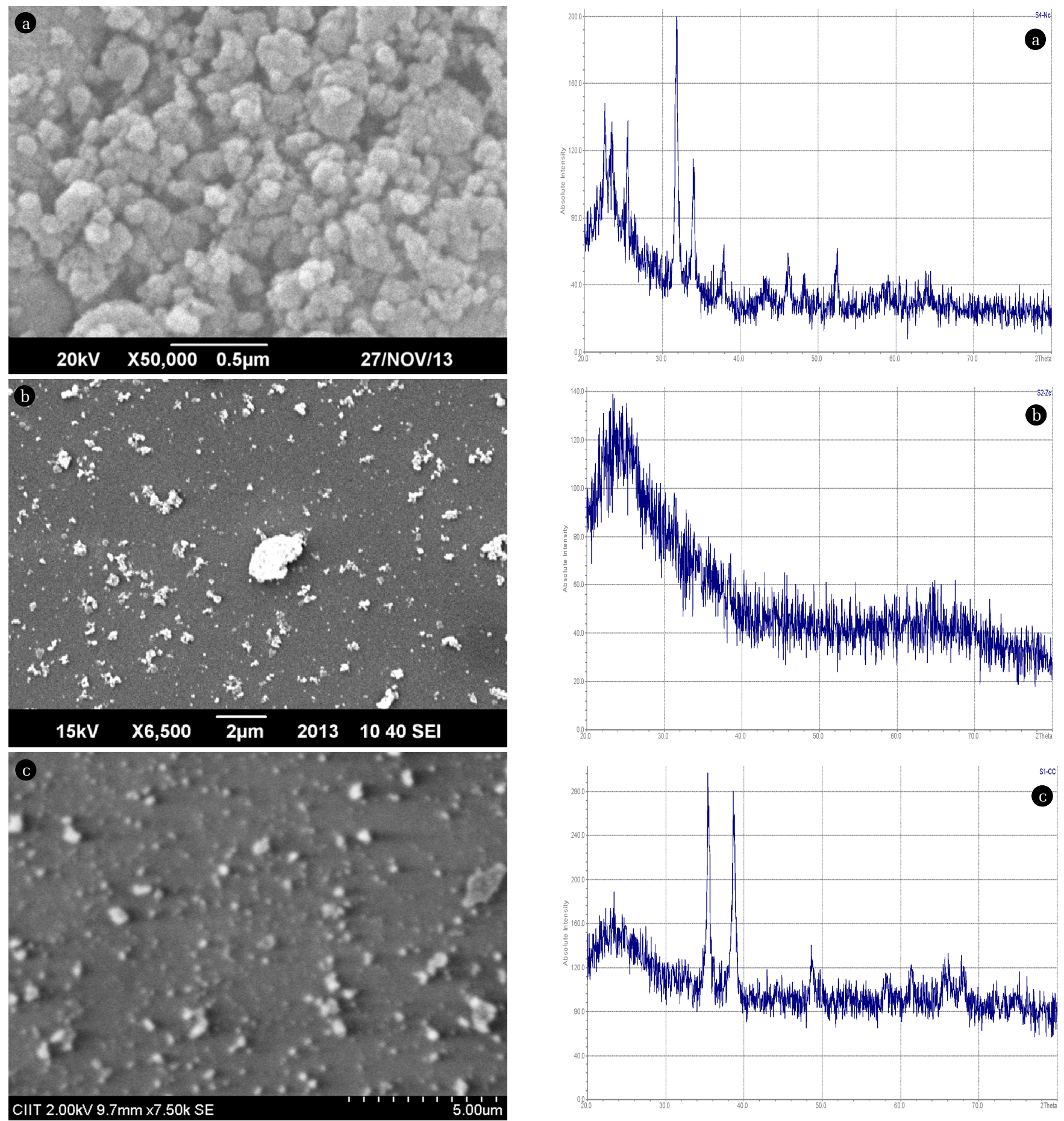

Fig. 2. Surface morphology of Silica doped (a) Si-Ni, (b) Si-Zn, and (c) $\mathrm{Si}-\mathrm{Cu}$ particles.

matrix. The peaks from $22.5^{\circ}$ to $25.2^{\circ}$ can be attributed to amorphous silica species. The strong peaks at $31.9^{\circ}$ and $34.0^{\circ}$ can be attributed to $\mathrm{Ni}$ species thus it confirms the incorporation of $\mathrm{Ni}$ in to $\mathrm{SiO}_{2}$ matrix Khafag et al. [21]. While the X-ray diffraction analysis of Zn showed amorphous geometry.

Fig. 3. X-ray diffraction analysis (XRD) spectra of silica doped (a) Si-Ni, (b) $\mathrm{Si}-\mathrm{Zn}$, and (c) Si-Cu particles.

The crystallite sizes of samples $\mathrm{Ni}$ and $\mathrm{Cu}$ were calculated by employing Debye-Scherrer equation,

$$
B(2 \theta)=K \lambda / L \cos \theta \text {. }
$$

The crystallite sizes of $\mathrm{Ni}$ and $\mathrm{Cu}$ turned out to be $12.3 \mathrm{~nm}$. 


\subsection{FTIR Analysis}

The synthesized nano sorbents were subjected to FTIR analysis as pellets. The spectrum was scanned over full range from 4,000 $\mathrm{cm}^{-1}$ to $400 \mathrm{~cm}^{-1}$. The peak around $468 \mathrm{~cm}^{-1}$ in zinc silica nanomaterials after calcination is ascribed to Zn-O stretching while peak at 1,089.82 $\mathrm{cm}^{-1}$ shows Si-O stretching (Fig. 4(b)), these peaks are comparable with those of $466 \mathrm{~cm}^{-1}$ and $1098 \mathrm{~cm}^{-1}$ reported in literature [22].
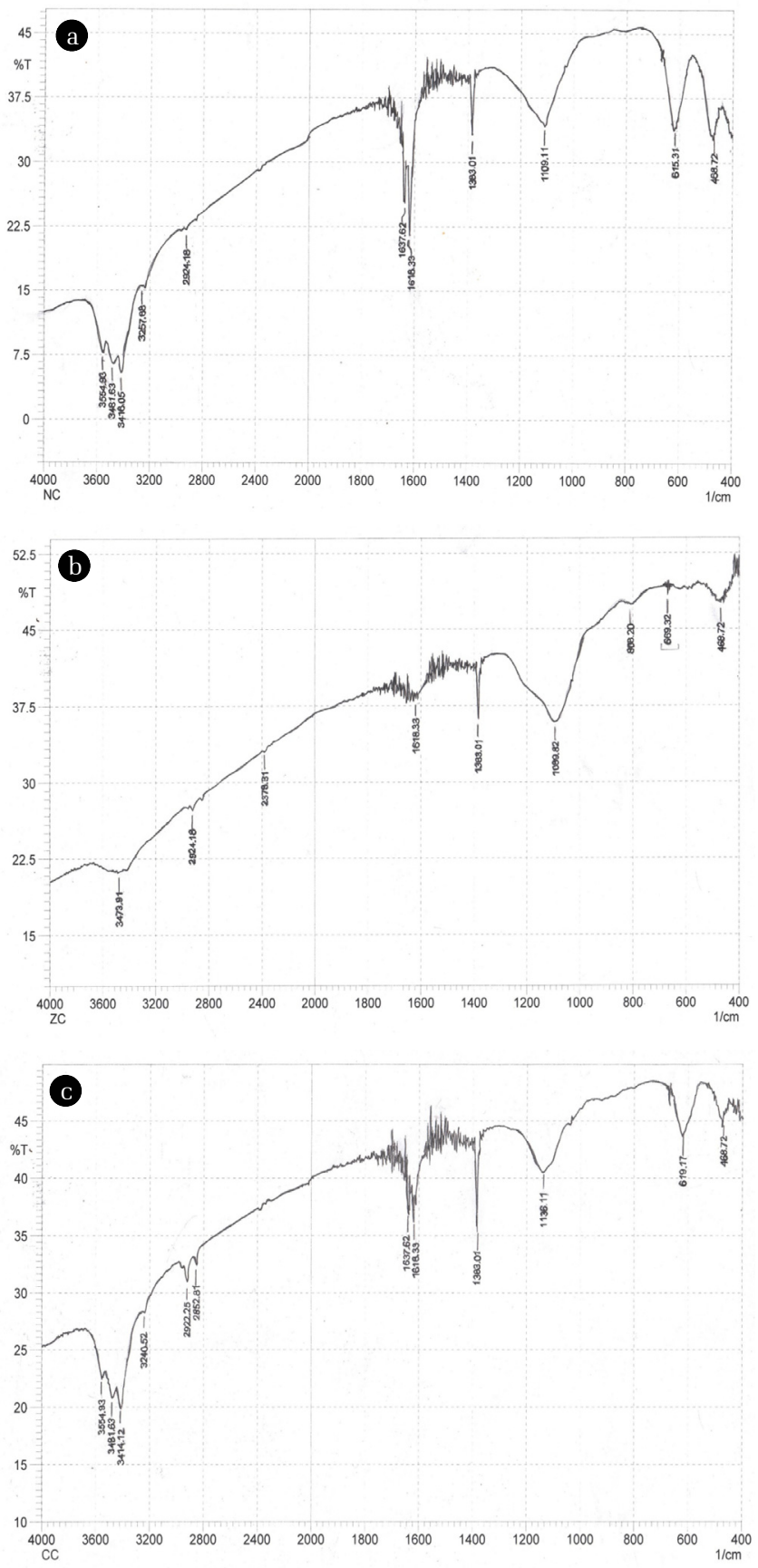

Fig. 4. Fourier transform infrared spectroscopy (FTIR) spectra of silica doped (a) Si-Ni, (b) Si-Zn, and (c) Si-Cu particles.
The presence of $\mathrm{Zn}-\mathrm{O}$ stretching at $468 \mathrm{~cm}^{-1}$ in the spectra is taken as indication of incorporation of metal into silica network. A weak peak at $808.32 \mathrm{~cm}^{-1}$ suggests polymerization of silica with oxygen, i.e., Si-O-Si symmetric vibration [23, 24]. The band recorded at $1,380 \mathrm{~cm}^{-1}$ is assigned to nitrates, carbonates as reported by Waseem et al. [25]. Absorption bands within range of $3,400-3,500 \mathrm{~cm}^{-1}$ are due to stretching vibrations of silanols, alcohols and hydroxyl groups. These bands show hydrogen bonding between silanol and hydroxyl groups. The bending vibrations of hydroxyl groups are reported to be present around 1,630-1,640 cm-1 [25].

In copper doped silica nanomaterials peak observed at 468.72 $\mathrm{cm}^{-1}$ is given to Si-O-Si bending vibrations. Peak at $617 \mathrm{~cm}^{-1}$ can be given to $\mathrm{Cu}-\mathrm{O}$ stretching vibration serving as indication of formation of new bonds within gel [26]. The vibrations of $\mathrm{Cu}-\mathrm{O}$ within range of $430-500 \mathrm{~cm}^{-1}$ can't be interpreted easily because of less concentration of copper and presence of very weak and overlapped peaks of silica masking Cu-O vibrations [27] (Fig. 4(c)).

$\mathrm{Ni}-\mathrm{O}$ band is observed at $468.32 \mathrm{~cm}^{-1}$ which is comparable to that mentioned in literature, i.e., $455 \mathrm{~cm}^{-1}$.

This position is also shared by bending $\mathrm{Si}-\mathrm{O}$ vibration. The peaks within $500-700 \mathrm{~cm}^{-1}$ region can be attributed to metal hydroxides (Fig. 4(a)) here the peak is found at $615 \mathrm{~cm}^{-1}$ [28].

IR spectra of adsorbed thiophene onto all silica based metal nanoparticles at room temperature show two bands in the range $1,600-1,300 \mathrm{~cm}^{-1}$. The bands at $1,462.09 \mathrm{~cm}^{-1}$ and $1,454 \mathrm{~cm}^{-1}$ obtained upon contact of Si metal nanoparticles at different time intervals can be described as $(\mathrm{C}=\mathrm{C})$ sym of thiophene adsorbed onto metal loaded Si nanomaterials via $\mathrm{S}$ atom. While band at $1,384.94 \mathrm{~cm}^{-1}$ can be ascribed to adsorbed thiophene as it shifts to lower frequency from $1,396 \mathrm{~cm}^{-1}$ by 12 to $19 \mathrm{~cm}^{-1}$ with time showing symmetric stretching vibration of $\mathrm{C}=\mathrm{C}$ in the fundamental ring [29]. This shift in wavenumbers of respective peak is caused by a decrease in the electron density of the entire thiophene ring implying that the ring of the adsorbed thiophene molecule is parallel to the surface of the adsorbent [30] that is, thiophene (soft Lewis base) is adsorbed onto metal ions (soft Lewis acids) loaded over silica by pi-electronic interactions.

The IR spectra of synthesized nanomaterials show peaks from $3,400-3,500 \mathrm{~cm}^{-1}$ (Fig. 4) corresponding to $\mathrm{OH}$ stretching vibrations of silanol groups. However these peaks disappear during the adsorbent-adsorbate interaction at room temperature. While the peaks of very low intensity at $2,800-2,900 \mathrm{~cm}^{-1}$ are enhanced remarkably corresponding to symmetric vibrations of $-\mathrm{CH}$. These peaks show hydrogen bonding between $-\mathrm{CH}$ and water or other glycerol hydroxyl groups [31].

The progress of reaction was examined by appearance and disappearance of peaks in spectra. Peaks observed at $2,860.53 \mathrm{~cm}^{-1}$ and $2,924.18 \mathrm{~cm}^{-1}$ correspond to $-\mathrm{CH}_{2}$ group vibrations and these results are comparable with those reported by Foresti et al. [32] and Sara et al. [33], i.e., bands between 2,980 and 2,850 $\mathrm{cm}^{-1}$ result due to stretching vibrations of $\mathrm{CH}_{2}$ groups close to a double bond $\left(\mathrm{CH}_{2}-\left(\mathrm{CH}=\mathrm{CH}_{2}\right)\right)$ or sulphur atom $\left(\mathrm{CH}_{2}-\mathrm{S}-\right)$. Appearance of peaks around 2,700 and $2,380 \mathrm{~cm}^{-1}$ in thiophene adsorption spectra of $\mathrm{Ni}$ and $\mathrm{Cu}$ indicate hydrogen-bonding type of interaction between $\mathrm{SH}$ functional groups in mercaptans and surface $\mathrm{SiOHAl}$ groups [30] implying opening of thiophene ring leading to alkenethiol 
species formation.

The peaks at $675,725,755,904,889 \mathrm{~cm}^{-1}$ correspond to out of plane vibrations of adsorbed benzothiophene. While the bands at $1,338,1,377,1,454,1,469 \mathrm{~cm}^{-1}$ point towards chemical transformation of benzothiophene. The presence of bands near 3,000 $\mathrm{cm}^{-1}$, i.e., 2,960, 2,964, 2,860 $\mathrm{cm}^{-1}$ indicates that the aromatic ring of benzothiophene remains intact while thiophene ring is opened by adsorption.

The bands observed around 3,150-3,200 $\mathrm{cm}^{-1}$ can be ascribed to aromatic/heterocyclic $\mathrm{C}-\mathrm{H}$ vibrations indicating the interaction of benzothiophene with the metal cations [34]. This is also supported by Yang et al. [35] as ring of thiophene derivatives interacts with transition metal cation through s-p electron contributions resulting in a stronger adsorption complex than other alkali and alkaline earth metals like $\mathrm{Na}^{+}$having no back donation of $\mathrm{d}$ electrons to the heterocyclic ring of the sulphur compounds

\subsection{GC-MS Analysis of Fuel}

The present investigation with GC-MS was undertaken for qualitative analysis of thiophenes in fuels. The commercial fuels of petrol and jet fuel were run on GC-MS using n-hexane as diluting solvent. Highest relative intensity is depicted by fragment $(\mathrm{m} / \mathrm{z}$ 18) in both fuels as a common feature. Presence of sulphur is qualitatively identified in petrol and jet fuel with relative intensity of $39.48 \%$ and $21.23 \%$, respectively. Retention time is noted as 34.4 and $12.8 \mathrm{~min}$ in respective order for petrol and jet fuel.

\subsection{Batch Experiment}

Batch experiments were conducted to assess the adsorptive potential of synthesized nano metal particles. Four sets of open batch as a function of time were conducted for the selective adsorption of thiophene and 1-benzothiophene as representatives of organo-sulphur compounds. The percentage removal was calculated for the sulphur compounds to determine the efficacy of synthesized nano-adsorbents.

\subsubsection{Effect of time}

The adsorbate adsorbent interaction was evaluated for $30 \mathrm{~min}$ with a regular interval of 2 min to determine the impact of contact time for the removal of thiophene and 1-benzothiophene by each silica based metal nanoparticle. The results are graphically presented in (Fig. 5).

It was generally observed that maximum adsorption occurs upon the first contact. This may be attributed to the fact that virgin adsorbent has more available sites providing space for rapid uptake of adsorbate. The continual uniform trend over the period of time significantly indicates strong interaction bond between nano-adsorbents and sulphur specie leading to chemisorption of thiophene and benzothiophene [36].

It is also noted that more than one equilibrium is established during the course of study. This initially identifies that each equilibrium is instantaneous and also indicates the heterogeneity of the system.

A striking consistent and optimal removal of thiophene and benzothiophene is generally observed for all metal nano-adsorbents. Removal efficiency of greater than $98 \%$ is evident over extended
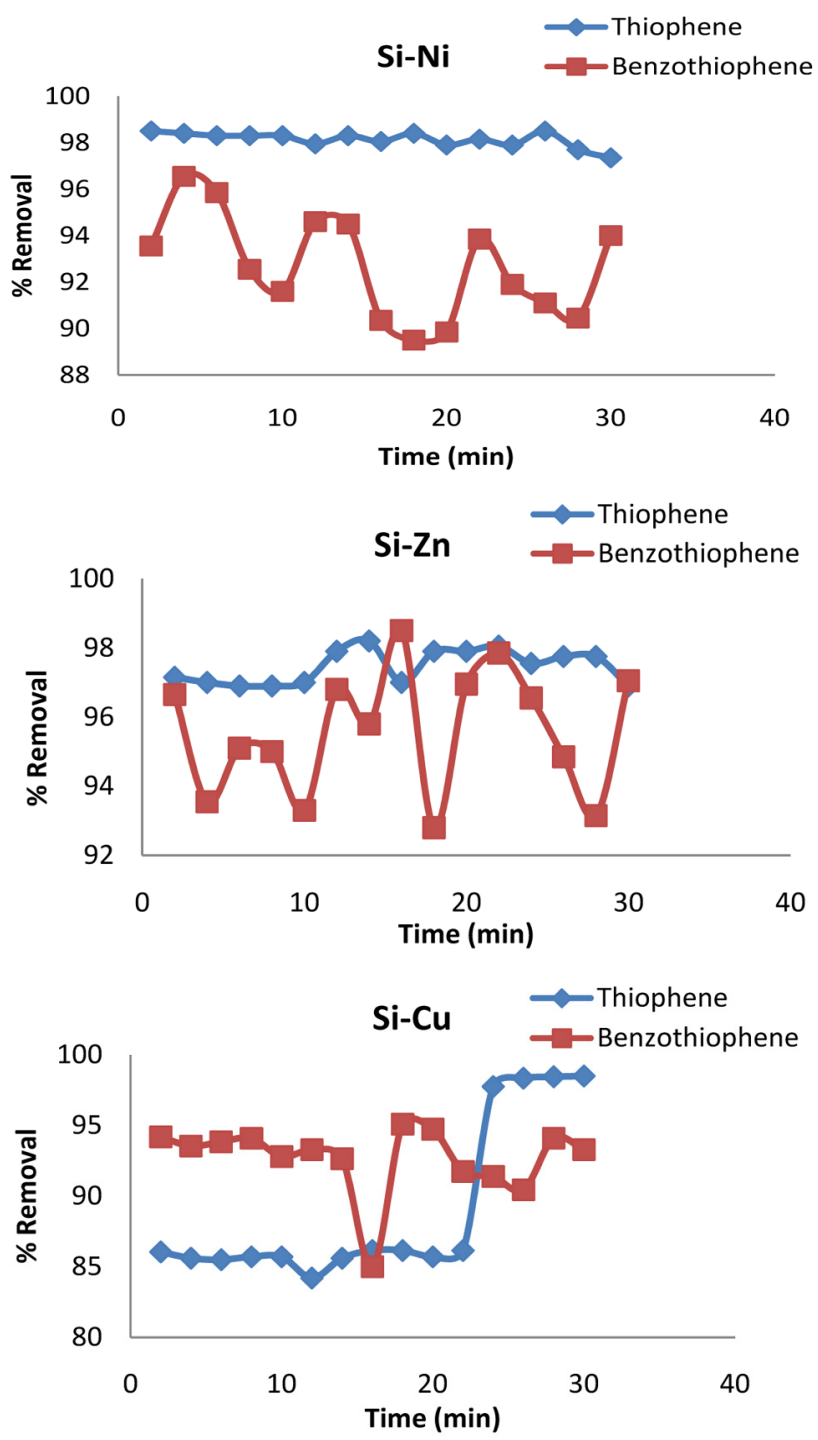

Fig. 5. Adsorption of thiophene and benzothiophene at silica doped metal particles as a function of time.

period of $30 \mathrm{~min}$. However, Cu-doped silica attained optimal efficiency earlier in 24 min (Fig. 5(c)). The maxima and minima in the graphs can be assigned to the adsorption and desorption process, respectively. Desorption can be explained on the basis of energy difference between the surface layers of adsorbent due to its heterogeneous nature. Greater activation energy for desorption of adsorbate is required for the system [4].

On comparison, it becomes evident that removal of 1-benzothiophene on nano-adsorbents shows a slightly lower removal and non-uniform trend than thiophene (see Fig. 5). This difference can be attributed to structural differences, molecular weight and density of both compounds. 1-benzothiophene being derivative of thiophene is larger and possesses high density $\left(1.15 \mathrm{~g} / \mathrm{cm}^{3}\right)$ and more stability towards reactions. Moreover, steric hindrance makes sulphur atom less accessible to the binding sites [37] for removal of 1-benzothiophene. 


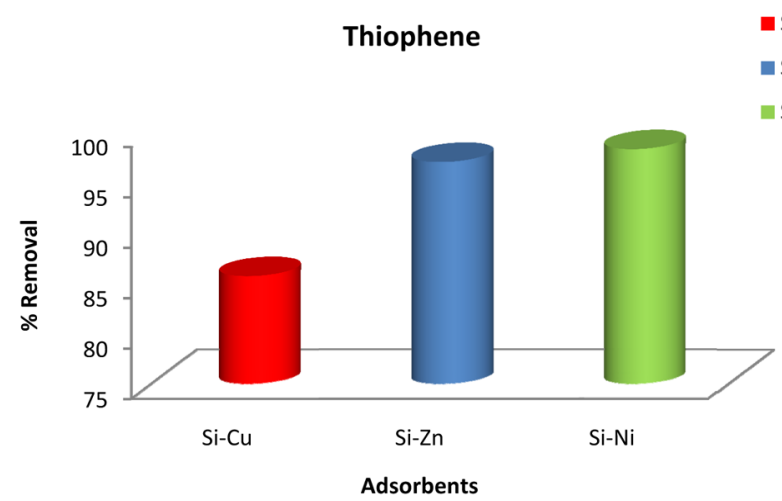

Benzothiophene

- Si-Cu

$\because \mathrm{Si}-\mathrm{Zn}$

$=\mathrm{Si}-\mathrm{Ni}$

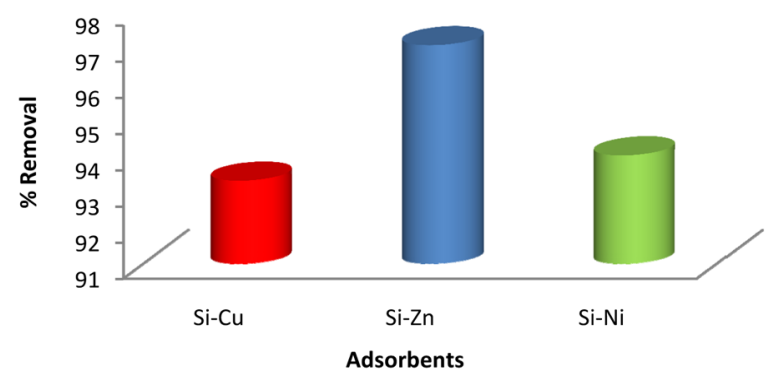

Fig 6. Adsorbents efficiency for removal of thiophene and benzothiophene.

\subsubsection{Effect of adsorbents}

The silica based metal nanoparticles were formed by sol-gel method which ensures easy and simple dealing and control over final product. The incorporation of metals in inorganic silica source TEOS provided much better anchorage to metals and improved their adsorptive capacity for removal of thiophene [38] and 1-benzothiophene from fuels. Furthermore, presence of hydroxyl groups on surface of silica based metal nanoparticles serve as acidic centers and facilitate binding of incoming species [39].

Silica in comparison to other reported substrates like $\mathrm{Al}_{2} \mathrm{O}_{3}$, $\mathrm{TiO}_{2}$ is weakly acidic because of the presence of silanol groups in it. Metals incorporation in silica matrix induces more acidity of varying binding strength [40], responsible for interaction of various species on adsorbents (Figs. 6 and 7).

Thiophene being a nucleophile has two electron pairs on it. One is parallel to its ring named $\sigma$-electron pair while the other is perpendicular called pi electrons. The metals with greater electropositive character (alkali, alkaline earth metals) are able to directly link with sulphur atom of thiophene ring through its $\sigma$-electrons [35]. However, the metals like $\mathrm{Cu}, \mathrm{Zn}$ (transition metals) are less electropositive in nature thus they bind with thiophene by their pi electrons following $\pi$-complexation [40]. Thiophene adopts different configurations for adsorption using both lone pairs of sulphur atom [2].

\subsubsection{Kinetic studies}

Different kinetic models including first order, pseudo-first-order, and pseudo-second-order and intra-particle diffusion model equa
Model fuel I $\quad 00 \mathrm{~min}$

- $30 \mathrm{~min}$

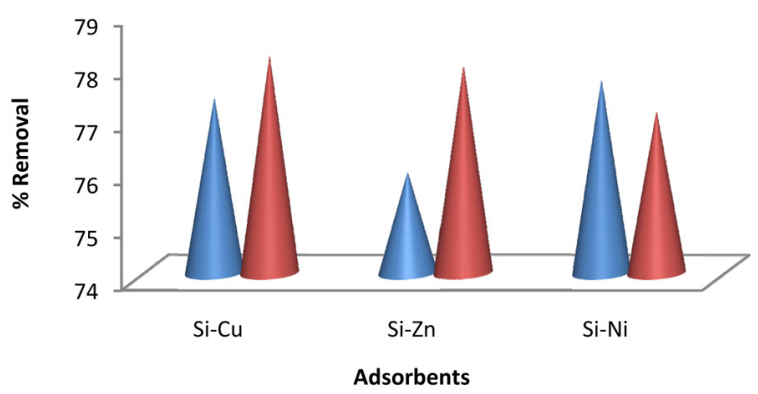

Model fuel II

a 0 min

- $30 \mathrm{~min}$

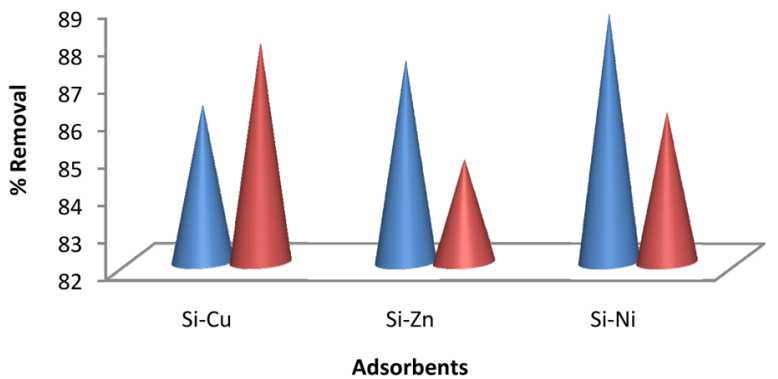

Fig. 7. Adsorption of thiophene and benzothiophene from model fuels i and ii by silica doped metal particles.
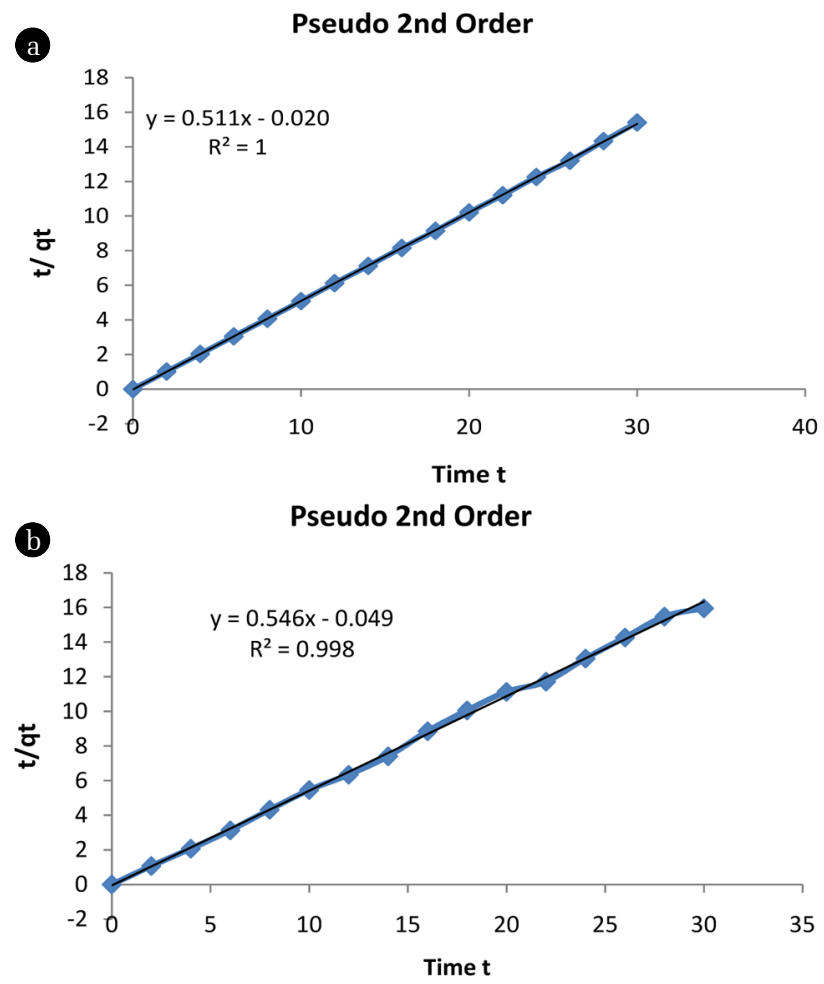

Fig 8. Kinetic model for adsorption of (a) thiophene and (b) benzothiophene. 
tions were applied to determine the mechanism involved in adsorption process. All the adsorption reactions followed pseudo-second-order kinetics for removal of thiophene and 1-benzothiophene (Fig. 8).

\subsubsection{Adsorption isotherms}

To explain the mechanism of adsorption of thiophene and 1-benzothiophene Freundlich, Langmuir, and Temkin isotherm models were applied on the data. The examination of correlation coefficient $\left(\mathrm{R}^{2}\right)$ showed that Freundlich isotherms model better explains the mechanism of adsorption of thiophene and 1-benzothiophene by synthesized nanomaterials (Fig. 9).

Sol-gel synthesis of silica metal nanoparticles provided better anchorage of metals in to silica matrix with Si to metal ratio 15:1. Thiophene is a soft Lewis base with two electron pairs on its sulphur atom. It has ability to form both sigma and pi bonds with metals. The metals $(\mathrm{Cu}, \mathrm{Ni}$, and $\mathrm{Zn})$ selected in this study lie on the borderline between hard and soft Lewis acids. Their doping on silica matrix creates acidic binding sites of varying strength. Depending on the electronegativity of the Lewis base and electropositive character of metals the affinity of base $\mathrm{N}>\mathrm{S}>\mathrm{O}$ varies towards acids thus nature of the bond changes from ionic to covalent.

In this study the bond responsible for attachment of thiophene on adsorbent surface is pi-bond. As the selected metals are serving as soft Lewis acids with relatively less electropositive character thus they form a weak bond through their d-electrons with thiophene and 1-benzothiophene. This soft Lewis acid base interaction

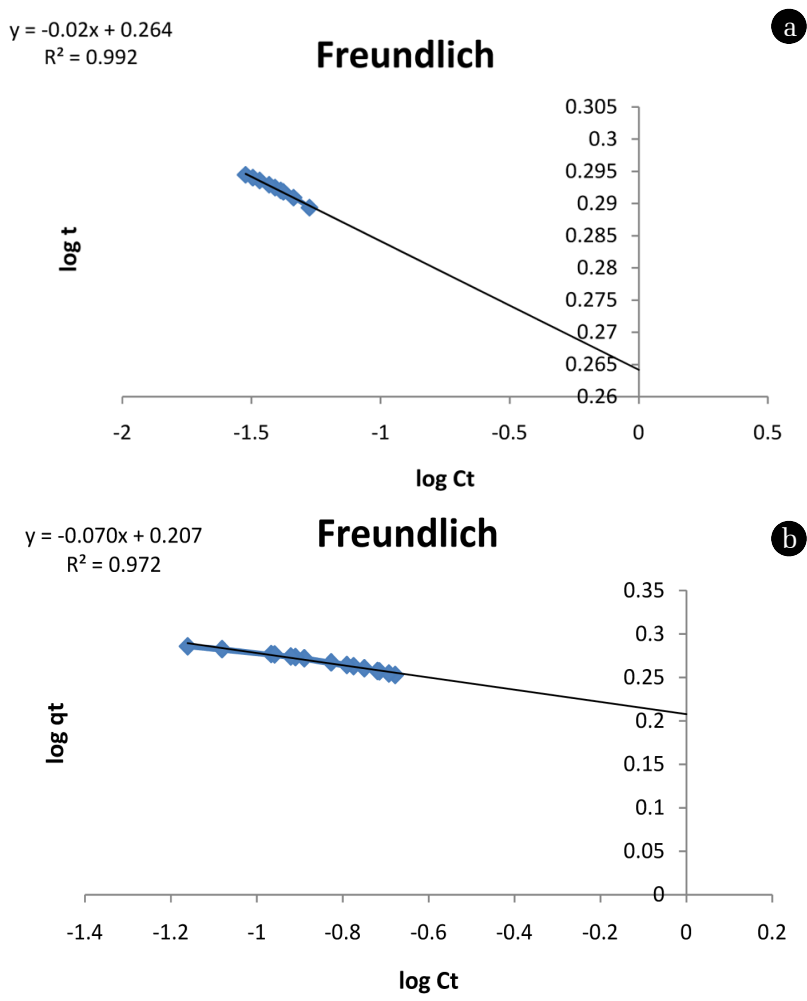

Fig. 9. Freundlich isotherm for adsorption of (a) thiophene and (b) benzothiophene. causes oligomerization of thiophenes leading to their removal from fuel stream at room temperature [41] as evidenced by appearance of $-\mathrm{CH}$ stretching vibrations at $2,800-2,900 \mathrm{~cm}^{-1}$ responsible for attachment of thiophene on silica binding sites [42]. These carbons result in bonding with metal adsorbent surface that lead to ring opening.

Furthermore, there is a prominent correlation between calcination temperature and Bronsted acidity of metal incorporated silica nanoparticles. Calcination results in decomposition of water molecules of metal salts and surface hydroxyl groups and convert them into isolated acidic $-\mathrm{OH}$ groups from vicinal and germinal groups as in $\mathrm{Ag}$ loaded $\mathrm{TiO}_{2}$ and $\mathrm{SiO}_{2}$ [43]. Proposed multilayered (Freundlich) adsorption mechanism follows $\pi$-complexation with pseudo-second-order kinetics [44].

\section{Conclusions}

The present study focused on greener synthesis of silica supported metal nanomaterials for desulphurization of fuels. Following conclusions can be drawn from the study: 1) glycerol as templating agent is attempted in the present investigation for the synthesis of metal doped silica particles. This provides a convenient and greener approach; 2) low metal to silica ratio suggests the $\pi$ -complexation mechanism for removal of refractory sulphur compounds; 3) polydispersity of nanoparticles allow adsorption of wide range of materials; 4) the nanomaterials followed the efficacy order as $\mathrm{Si}-\mathrm{Ni}>\mathrm{Si}-\mathrm{Zn}>\mathrm{Si}-\mathrm{Cu}$ based adsorbents; and 5) the proposed multilayered (Freundlich) adsorption mechanism follows $\pi$-complexation with pseudo-secnd-order kinetics.

\section{References}

1. Ma X, Sprague M, Sun L, Song C. Deep desulfurization of diesel fuels by a novel integrated approach. Pittsburgh: National Energy Technology Lab.; 2002.

2. Song C. An overview of new approaches to deep desulfurization for ultra-clean gasoline, diesel fuel and jet fuel. Catal. Today 2003;86:211-263.

3. Srivastava VC. An evaluation of desulfurization technologies for sulfur removal from liquid fuels. RSC Adv. 2012;2:759-783.

4. Yu M, Li Z, Xi H, Xia Q, Wang S. Effect of textural property of coconut shell-based activated carbon on desorption activation energy of benzothiophene. Front. Chem. Eng. China 2008;2: 269-275.

5. Muzic M, Sertic-Bionda K, Gomzi Z. Kinetic and statistical studies of adsorptive desulfurization of diesel fuel on commercial activated carbons. Chem. Eng. Technol. 2008;31:355-364.

6. Kim CG. Adsorption behaviour of thiophene derivatives on soil materials. Environ. Eng. Res. 2002;7: 207-217.

7. Kobayashi M, Flytzani-Stephanopoulos M. Reduction and sulfidation kinetics of cerium oxide and cu-modified cerium oxide. Ind. Eng. Chem. Res. 2002;41:3115-3123.

8. Hernández-Maldonado AJ, Yang RT. New sorbents for desulfurization of diesel fuels via $\pi$-complexation. AIChE J. 
2004;50:791-801.

9. Lu AH, Salabas EL, Schüth F. Magnetic nanoparticles: synthesis, protection, functionalization, and application. Angew. Chem. Int. Ed. Engl. 2007;46:1222-1244.

10. Anedda R, Cannas C, Musinu A, Pinna G, Piccaluga G, Casu M. A two-stage citric acid-sol/gel synthesis of $\mathrm{ZnO} / \mathrm{SiO}_{2}$ nanocomposites: study of precursors and final products. J. Nanopart. Res. 2008;10:107-120.

11. Chao MC, Lin HP, Mou CY, Cheng BW, Cheng CF. Synthesis of nano-sized mesoporous silicas with metal incorporation. Catal. Today 23004;97:81-87.

12. Yu LY, Xu ZL, Shen HM, Yang H. Preparation and characterization of PVDF-SiO2 composite hollow fiber UF membrane by sol-gel method. J. Memb. Sci. 2009;337:257-265.

13. Lai Y, Yin W, Liu J, Xi R, Zhan J. One-pot green synthesis and bioapplication ofl-arginine-capped superparamagnetic $\mathrm{Fe}_{3} \mathrm{O}_{4}$ nanoparticles. Nanoscale Res. Lett. 2009;5:302-307.

14. Seredych M, Mabayoje O, Koleśnik MM, Krstić V, Bandosz TJ. Zinc (hydr)oxide/graphite based-phase composites: effect of the carbonaceous phase on surface properties and enhancement in electrical conductivity. J. Mater. Chem. 2012;22:79707978.

15. Dai W, Zhou Y, Wang S, Sub W, Sun Y, Zhou L. Desulfurization of transportation fuels targeting at removal of thiophene/ benzothiophene. Fuel Process. Technol. 2008;89:749-755.

16. Lawal OA, Oyedeji AO. Chemical composition of the essential oils of Cyperus rotundus L. from South Africa. Molecules 2009;14:2909-2917.

17. Liu X, Chun CM, Aksay IA, Shih WH. Synthesis of mesostructured nickel oxide with silica. Ind. Eng. Chem. Res. 2000;39: 684-692.

18. Das D, Nath BC, Phukon P, Dolui SK. Synthesis and evaluation of antioxidant and antibacterial behavior of $\mathrm{CuO}$ nanoparticles. Colloids Surf. B Biointerfaces 2013;101:430-433.

19. Thekkae Padil VV, Černík M. Green synthesis of copper oxide nanoparticles using gum karaya as a biotemplate and their antibacterial application. Int. J. Nanomedicine 2013;8:889-898.

20. Prakasham RS, Devi GS, Rao CS, Sivakumar VS, Sathish T, Sarma PN. Nickel-impregnated silica nanoparticle synthesis and their evaluation for biocatalyst immobilization. Appl. Biochem. Biotechnol. 2010;160:1888-1895.

21. Khafag MG, Mahmoud HH, Ashiry A, Israr MQ, Battisha IK Characterization of nano-composite $\mathrm{Ni}$ dispersed over a silica matrix prepared by a modified sol-gel technique. Aust. J. Basic Appl. Sci. 2011;5:287-295.

22. Roy A, Polarz S, Rabe S, et al. First preparation of nanocrystalline zinc silicate by chemical vapor synthesis using an organometallic single-source precursor. Chemistry 2004;10:1565-1575.

23. Kong DY, Yu M, Lin CK, Liu XM, Lin J, Fang J. Sol-gel synthesis and characterization of $\mathrm{Zn}_{2} \mathrm{SiO}_{4}: \mathrm{Mn} @ \mathrm{SiO}_{2}$ spherical core-shell particles. J. Electrochem. Soc. 2005;152:H146-151.

24. Shokri B, Firouzjah MA, Hosseini SI. FTIR analysis of silicon dioxide thin film deposited by metal organic-based PECVD. In: Proceedings of 19th International Symposium on Plasma Chemistry Society; 2009 Jul 26-31; Bochum, Germany. p. 26-31. 25. Waseem M, Mustafa S, Naeem A, Shah KH, Ihsan-ul-Haque
IS. Synthesis and characterization of silica gel by sol-gel method. J. Pak. Mater. Soc. 2009;3:19-21.

26. Cordoba G, Arroyo R, Fierro JL, Viniegr M. Study of xerogel-glass transition of $\mathrm{CuO} / \mathrm{SiO}_{2}$. J. Solid State Chem. 1996;123;93-99.

27. Fixman EM, Abello MC, Gorriz OF, Arrúa LA. Preparation of $\mathrm{Cu} / \mathrm{SiO}_{2}$ catalysts with and without tartaric acid as template via a sol-gel process: characterization and evaluation in the methanol partial oxidation. Appl. Catal. A Gen. 2007;319:111118.

28. Battishaa IK, Afifya $\mathrm{HH}$, Ibrahim M. Synthesis of $\mathrm{Fe}_{2} \mathrm{O}_{3}$ concentrations and sintering temperature on FTIR and magnetic susceptibility measured from 4 to $300 \mathrm{~K}$ of monolith silica gel prepared by sol-gel technique. J. Magn. Magn. Mater. 2006;306 211-217.

29. Tian F, Wu W, Jiang Z, et al. The study of thiophene adsorption onto La(III)-exchanged zeolite NaY by FT-IR spectroscopy. $J$. Colloid Interface Sci. 2006;301:395-401.

30. Garcia CL, Lercher JA. Adsorption and surface reactions of thiophene on ZSM 5 zeolites. J. Phys. Chem. 1992;96:2669-2675.

31. Dashnau JL, Nucci NV, Sharp KA, Vanderkooi JM. Hydrogen bonding and the cryoprotective properties of glycerol/water mixtures. J. Phys. Chem. B. 2006;110:13670-13677.

32. Foresti E, Fracasso G, Lanzi M, et al. New thiophene monolayer-protected copper nanoparticles: synthesis and chemical-physical characterization. J. Nanomater. 2008;2008:1-6.

33. Sara YY, Garcia-Martinez J, Li W, Meitzner GD, Iglesia E. Kinetic, infrared, and X-ray absorption studies of adsorption, desorption, and reactions of thiophene on H-ZSM5 and Co/H-ZSM5. Phys. Chem. Chem. Phys. 2002;4:1241-1251.

34. Jiang M, Ng FT. Adsorption of benzothiophene on Y zeolites investigated by infrared spectroscopy and flow calorimetry. Catal. Today 2006;116:530-536.

35. Yang X, Erickson LE, Hohn KL, Jeevanandam P, Klabunde KJ. Sol-gel Cu-Al2O3 adsorbents for selective adsorption of thiophene out of hydrocarbon. Ind. Eng. Chem. Res. 2006;45: 6169-6174.

36. Blanco-Brieva G, Campos-Martin JM, Al-Zahrani SM, Fierro JL. Removal of refractory organic sulfur compounds in fossil fuels using MOF sorbents. Global Nest J. 2010;12:296-304.

37. Li W, Xing J, Xiong X, Huang J, Liu H. Feasibility study on the integration of adsorption/bioregeneration of $\pi$-complexation adsorbent for desulfurization. Ind. Eng. Chem. Res. 2006;45:28452849.

38. Xue M, Wen P, Chitrakar R, Ooi K, Feng Q. Screening of inorganic adsorbents for selective adsorption of thiophene from model gasoline. Sep. Sci. Technol. 2012;47:1926-1936.

39. Hussain AH, Tatarchuk BJ. Adsorptive desulfurization of hydrocarbon fuels by $\mathrm{Ag} / \mathrm{TiO}_{\mathrm{x}}-\mathrm{Al}_{2} \mathrm{O}_{3}$ adsorbents: mechanism of sulfur adsorption at ambient conditions. In: Proceedings of the 2012 AlChE Annual Meeting; 2012 Oct 28-Nov 2; Pittsburgh, PA.

40. Kosslick H, Lischke G, Landmesser H, Parlitz B, Storek W, Fricke R. Acidity and catalytic behavior of substituted MCM-48. J. Catal. 1998;176:102-114.

41. Fuentes-Perujo D, Santamaría-González J, Mérida-Robles J, et al. Evaluation of the acid properties of porous zirconium-doped 
and undoped silica materials. J. Solid State Chem. 2006;179: 2182-2189.

42. Vilarrasa-García E, Azevedo DC, Braos-García P, et al. Synthesis and characterization of metal-supported mesoporous silicas applied to the adsorption of benzothiophene. Adsorpt. Sci. Technol. 2011;29:691-704.
43. Nair S, Shahadat Hussain AH, Tatarchuk BJ. The role of surface acidity in adsorption of aromatic sulfur heterocycles from fuels. Fuel 2013;105:695-704.

44. Tabassum N, Rafique U, Balkhair KS, Ashraf MA. Chemodynamics of methyl parathion and ethyl parathion: adsorption models for sustainable agriculture. BioMed Res. Int. 2014;2014:831989. 